\title{
Repeated-stimulus superiority and inferiority effects in the identification of letters and digits
}

\author{
ROBERT W. PROCTOR and GENE W. FOBER \\ Auburn University, Auburn, Alabama
}

\begin{abstract}
When a target stimulus in a predesignated location is identified by a keypress response, responses are slightly faster if noise stimuli in adjacent locations are identical to the target than if they are a different stimulus assigned to the same response (a repeated-stimulus superiority effect). An exception to this result has been found in experiments that used randomly intermixed letter and digit stimuli. These experiments showed slower responding for identical noise than for nonidentical, response-compatible noise (a repeated-stimulus inferiority effect). The present study investigated these phenomena in three experiments. Experiments 1 and 2 established that both the superiority and inferiority effects can be obtained in the same experiment. They also provided evidence that the repeated-stimulus inferiority effect is a function of the intermixing of letters and digits and not of the larger target-set size that has been used for mixed lists. Experiment 3 demonstrated that, with unmixed presentation, the repeated-stimulus superiority effect is enhanced by an increase in the number of stimuli assigned to each response. The experiments are consistent with accounts that attribute the repeated-stimulus superiority effect to competition that occurs when different internal recognition responses are activated. Moreover, the experiments suggest that the repeated-stimulus inferiority effect has its basis in processes that occur subsequent to feature extraction.
\end{abstract}

The manner in which multiple stimuli interact has been a persisting issue in the study of visual information processing (e.g., Crosland, 1931; C. W. Eriksen, 1966; Estes, 1982). A target-identification task that has proved to be useful in examining this issue was developed by B. A. Eriksen \& C. W. Eriksen (1974). For the task, stimuli (usually letters) are assigned to either of two responses (in some experiments, movements of a lever to the left or right; in others, keypress responses with the left or right index finger). Subjects are required to identify a single stimulus (the target) that is presented in a known location (most often, foveally) by making the assigned response. On most trials, noise stimuli are presented simultaneously with the target in locations that are adjacent to it. The level at which the noise influences processing can be determined by varying the relationship between the target and noise.

Experiments that utilized the Eriksens' targetidentification task have shown that the noise influences processing primarily through response competition. That is, substantial interference in responding results when the

We would like to thank Mei-Shio Jang for programming the experiments and Ladana Lindsey, Hunter Peak, Jeff Smalley, Connie Vitteri, and Beth Waite for assistance in collecting the data. We also appreciate the helpful comments on an earlier version of the manuscript that were provided by LeLaine Dornier, Georgann Lucariello, Gil Reeve, and LaVerne Worthy. G. W. Fober is associated with the Motor Behavior Center at Auburn University. Requests for reprints should be sent to Robert W. Proctor, Department of Psychology, Auburn University, Auburn, AL 36849. noise is a potential target assigned to the opposing response from that indicated by the actual target for the trial (incompatible noise; e.g., B. A. Eriksen \& C. W. Eriksen, 1974; C. W. Eriksen \& B. A. Eriksen, 1979; C. W. Eriksen \& Schultz, 1979; Grice, Boroughs, \& Canham, 1984; Grice, Canham, \& Schafer, 1982; Taylor, 1977). Similar competition also seems to occur between internal recognition, or naming, responses (C. W. Eriksen \& Schultz, 1979; Proctor, 1981). When two stimuli are assigned to each of two possible responses (e.g., D,N to one response and $R, S$ to another), responses tend to be slightly slower when the noise is the alternative stimulus assigned to the same response as the target (compatible noise) than when the noise is identical to the target (identical noise; B. A. Eriksen \& C. W. Eriksen, 1974; C. W. Eriksen \& B. A. Eriksen, 1979; C. W. Eriksen \& Schultz, 1979; Grice \& Gwynne, 1985; Taylor, 1977; Yeh \& C. W. Eriksen, 1984). This small repeatedstimulus superiority effect apparently is due to interference from the competing recognition responses signaled in the compatible-noise condition, because the identicalnoise condition shows no advantage over a neutral-noise condition or over presentation of the target alone (B. A. Eriksen \& C. W. Eriksen, 1974; C. W. Eriksen \& B. A. Eriksen, 1979; C. W. Eriksen \& Schultz, 1979; Grice et al., 1984; Grice \& Gwynne, 1985; Krueger \& Shapiro, 1980; Proctor, 1981).

An exception to the repeated-stimulus superiority effect was reported by Flowers and Wilcox (1982) and LaBerge (1981) for experiments that used both letter and 
digit stimuli. In their experiments, two letters and two digits were assigned to each of two keypress responses (e.g., Flowers \& Wilcox assigned the set D,N,3,7 to one response and the set $R, S, 4,5$ to the other). The target and noise stimuli on any given trial could both be letters, both be digits, or a letter-digit (digit-letter) combination. Contrary to the studies described in the previous paragraph, which did not mix letters and digits, the experiments that did mix the two stimulus types found a repeated-stimulus inferiority effect. That is, responses were slower when the noise was identical to the target than when it was the alternative stimulus from the same category that was assigned to the same response as was the target (samecategory-compatible noise; e.g., target $\mathrm{D}$, noise $\mathrm{N}$ ). ${ }^{1}$ Responses for the same-category-compatible trials also were faster than those for neutral-noise trials, suggesting that response-compatible noise facilitates processing (Flowers \& Wilcox, 1982). Flowers and Wilcox proposed that this facilitation is counteracted by feature-specific inhibition that occurs during perceptual encoding when the target and noise are identical (see Krueger \& Shapiro, 1980 , and Miller, 1982, for similar speculations).

Although the results obtained by Flowers and Wilcox (1982) and LaBerge (1981) are consistent with a featurespecific inhibition explanation, there are at least two reasons to doubt this interpretation. First, evidence for feature-specific inhibition in other tasks is minimal (but see La Heij \& van der Heijden, 1983). The strongest support for feature-specific inhibition was a repeated-letter inferiority effect obtained by Bjork and Murray (1977) in a postcuing task. However, subsequent studies have shown convincingly that their results were due to experimental artifacts (C. W. Eriksen, Morris, Yeh, O'Hara, \& Durst, 1981; Estes, 1982; Santee \& Egeth, 1980,1982 ). Second, as noted previously, the studies that used stimuli from only a single alphanumeric category have found identical-noise trials to have a small advantage relative to compatible-noise trials, rather than a disadvantage (B. A. Eriksen \& C. W. Eriksen, 1974; C. W. Eriksen \& B. A. Eriksen, 1979; Grice \& Gwynne, 1985; Taylor, 1977; Yeh \& C. W. Eriksen, 1984). If the repeated-stimulus inferiority effect was due to featural interactions in perceptual encoding, the effect should occur regardless of whether categories are mixed. Therefore, if letters and digits must be mixed to obtain the repeated-stimulus inferiority effect, the effect is likely to be due to some process other than feature-specific inhibition.

Although the repeated-stimulus superiority and inferiority effects both have important implications for models of visual information processing, neither the validity of the respective effects nor the conditions under which each occurs has been established clearly. The small superiority effect has been obtained in most experiments for which the stimuli were from a single alphanumeric category, but in some cases the effect has been nonsignificant (B. A. Eriksen \& C. W. Eriksen, 1974) or absent (Miller, 1982). The inferiority effect, which has been found when letters and digits were mixed, was reported in two experiments
(Flowers \& Wilcox, 1982; LaBerge, 1981) that differed in several other respects from the experiments that did not mix letters and digits. Moreover, one of the researchers who reported the repeated-stimulus inferiority effect (LaBerge, 1981) noted that it was not obtained in other, unreported, experiments from his laboratory. Because of the relative uncertainty surrounding the repeatedstimulus superiority and inferiority effects, the present experiments were designed (1) to evaluate the validity of each effect and (2) to delineate the conditions under which each occurs.

\section{EXPERIMENT 1}

The purpose of Experiment 1 was to determine whether the discrepant pattern of results found previously when letters and digits were mixed, rather than unmixed, could be obtained within a single experiment. The methods for mixed presentation followed closely those of Flowers and Wilcox's (1982) Experiment 3, which obtained a repeated-stimulus inferiority effect. Two letters and two digits were assigned to each of two keypress responses, and subjects received a practice session to learn the assignments. Subsequently, three test sessions were conducted in which the letters and digits were mixed, only the letters were used, and only the digits were used, respectively.

\section{Method}

Apparatus and Stimuli. Stimuli were presented on the display screen of a Radio Shack TRS-80 Model 4 microcomputer. The stimuli were white on a dark background. The viewing distance was not controlled, but was approximately $50 \mathrm{~cm}$. Responses were made by pressing either the $\mathrm{Z}$ or ? key on the computer's keyboard with the left or right index finger, respectively. All timing was controlled by the computer, with responses measured to the nearest millisecond.

The stimuli were four uppercase letters (D, N, R, and S) and four digits $(3,4,5$, and 7$)$, which were divided into two sets (D,N,3,7, and $R, S, 4,5$ ). The former set was assigned to the left response and the latter set to the right response for half of the subjects; the assignment was reversed for the other half. The stimulus sets and assignment procedures were the same as those used by Flowers and Wilcox (1982).

Each display contained a target stimulus flanked by two noise stimuli, with each stimulus subtending a visual angle of approximately $.40^{\circ}$ wide and $.63^{\circ}$ high as viewed by the subject. The target was displayed in a fixed location approximately $.68^{\circ}$ above an asterisk that served as a fixation spot, and the separation between the target and the noise stimuli was approximately $.40^{\circ}$. Each trial began with a $250-\mathrm{msec}$ offset of the fixation spot. Two-hundred and fifty milliseconds after the fixation spot reappeared, the target and noise stimuli were presented simultaneously and remained in view until the subject responded. After a 1-sec interval, the next trial sequence began.

There were six conditions based on the relationship between the target and noise. For the neutral-noise condition, the noise consisted of asterisks (e.g., * $\mathrm{R}^{*} ;{ }^{*} 4^{*}$ ). For all other noise conditions, the noise stimuli were members of the target set. Identical noise consisted of stimuli that were the same as the target (e.g., RRR; 444); same-category-compatible noise consisted of the other member from the same category as the target that was assigned to the same response (e.g., SRS; 545); same-category-incompatible noise consisted of a member from the same category as the target that was 
assigned to the opposite response (e.g., DRD; 343); differentcategory-compatible noise consisted of a member from the category opposite to that of the target that was assigned to the same response (e.g., 4R4; R4R); and different-category-incompatible noise consisted of a member from the category opposite to that of the target that was assigned to the opposite response (e.g., 3R3; D4D). The latter two noise conditions were applicable only when letters and digits were mixed within lists.

Lists of 144 trials were constructed for which all stimuli were from a single category (unmixed lists) or for which stimuli from both categories were intermixed (mixed lists). The mixed lists included 16 trials each from the neutral, identical, and samecategory-compatible conditions and 32 trials each from the differentcategory-compatible, same-category-incompatible, and differentcategory-incompatible conditions. These proportions of trials were the same as those used by Flowers and Wilcox (1982) and represent an equal number for all combinations of the specific target and noise stimuli. For the unmixed lists, the different-category conditions were inapplicable, so they were collapsed into the applicable conditions to yield 32 trials each for the identical and same-category-compatible conditions, 64 trials for the same-category-incompatible condition, and 16 trials for the neutral condition.

Four different mixed lists of 144 trials were constructed, with the constraint that all possible target-noise combinations appear equally often in both halves of each list. Four unmixed lists of letters and four unmixed lists of digits were constructed by replacing the letters (digits) in each mixed list, following the same constraint used in constructing the mixed lists.

Subjects and Procedure. The subjects were 24 students at $\mathrm{Au}-$ burn University who were enrolled in an introductory psychology course. Each subject participated in four sessions for extra credit toward his or her course grade. Data from five additional subjects were excluded because in at least one session either they responded incorrectly on over $10 \%$ of the trials or their RTs exceeded $1 \mathrm{sec}$ on over $10 \%$ of the trials.

The first session was a practice session intended to familiarize the subjects with the stimulus-response assignments. The session was conducted with a mixed list, and the particular assignments learned by the subject were used in the subsequent test sessions. At the beginning of the practice session, each subject read written instructions that explained the task and assigned two letters and two digits to each response. The instructions indicated that the target stimulus would occur in the location immediately above fixation, with irrelevant noise stimuli presented simultaneously in adjacent locations. The instructions stressed that responding to the target was to be as fast and accurate as possible. A block of 18 warm-up trials then was presented, followed by a set of 144 trials, with the trials involving mixed presentation of letters and digits.

Three test sessions were conducted subsequently, with one session devoted to a mixed list, another to an unmixed list of only letters, and another to an unmixed list of only digits. The order of these sessions was counterbalanced across subjects. In each session, the particular type of trials that would occur was explained to the subject, and 18 warm-up trials then were presented. These were followed by the 144-trial test list. The same stimulus-response assignments used by a subject in the practice session were used throughout the three test sessions, although only the letter or digit subsets were involved when presentations were unmixed.

\section{Results and Discussion}

Mean reaction times (RTs) for correct responses and proportions of errors were obtained for each subject as a function of presentation type (unmixed letters, unmixed digits, and mixed letters and digits) and target-noise relationship. Because the effect of target-noise relationship was relatively similar for letters and digits when presentation was unmixed, and because the amount of data obtained with mixed presentation was insufficient for an ac- curate comparison of the two categories, the data were collapsed across alphanumeric category (similar procedures were followed in Experiments 2 and 3). The group means for the respective target-noise conditions are shown in Table 1 as a function of mixed versus unmixed presentation.

Target-noise conditions common to mixed and unmixed lists. Separate two-factor analyses of variance were conducted on the RT and error data for the target-noise conditions that occurred in both the mixed and unmixed lists (i.e., the identical, same-category-compatible, samecategory-incompatible, and neutral conditions). These analyses showed that subjects were slower at responding to the mixed lists $(M=544 \mathrm{msec})$ than to the unmixed lists $(\mathrm{M}=504 \mathrm{msec})[\mathrm{F}(1,23)=13.9, \mathrm{p}<.002]$, with the error rates being similar $(\mathrm{Ms}=.032$ and .025 for the mixed and unmixed lists, respectively) $(F<1.0)$. The slightly greater difficulty of the mixed lists reflects primarily the greater number of potential targets for those lists (see Experiment 3).

Both the RT and error data also showed main effects for target-noise relationship $[F s(3,69) \geq 8.15$, ps $<$ .001]. As is found customarily (e.g., B. A. Eriksen \& C. W. Eriksen, 1974), the slowest and least accurate responses were obtained for the same-categoryincompatible trials ( $\mathrm{Ms}=539 \mathrm{msec}$ and .046). The samecategory-compatible trials showed the fastest responses $(\mathrm{M}=512 \mathrm{msec})$, with accuracy also being relatively high (error rate $=.023$ ). The identical and neutral trials fell intermediate to the other conditions on RTs $(\mathrm{Ms}=524$ and $522 \mathrm{msec}$, respectively). The error rate for the identical relationship also was intermediate $(M=.034)$, although the neutral relationship showed the fewest errors $(\mathrm{M}=.014)$.

Most importantly, a presentation $\times$ relationship interaction was evident in the RT data $[\mathrm{F}(3,69)=5.00$, $\mathrm{p}<$ $.005]$, as was a similar trend in the error data $[F(3,69)$ $=2.47, \mathrm{p}=.069]$. The interaction in the RT data occurred primarily because the same-category compatible and incompatible trials showed smaller increases from unmixed to mixed presentation ( 24 and $32 \mathrm{msec}$, respectively) than did the identical and neutral trials (57 and $46 \mathrm{msec}$, respectively). When only the identical and same-

Table 1

Mean Reaction Times (in Milliseconds) and Proportions of Errors for Experiment 1 as a Function of Presentation Method (Mixed, Unmixed) and Target-Noise Relationship

\begin{tabular}{lcccccc}
\hline & \multicolumn{7}{c}{ Target-Noise Relationship } \\
\cline { 2 - 7 } Measure & I & SC & SI & N & DC & DI \\
\hline Reaction Time & 495 & 500 & 523 & 499 & & \\
Error Rate & .020 & .019 & .047 & .014 & & \\
& \multicolumn{7}{c}{ Mixed } \\
Reaction Time & 552 & 524 & 555 & 545 & 546 & 560 \\
Error Rate & .047 & .026 & .044 & .013 & .029 & .043 \\
\hline
\end{tabular}

Note-I = identical; $S C=$ same-category-compatible; $S I=$ samecategory-incompatible; $N=$ neutral; $D C=$ differentcategory-compatible, and $D I=$ different-category-incompatible. 
category-compatible trials were analyzed, the presentation $\times$ relationship interaction still was significant $[F(1,23)=13.48, p<.002]$. Responses were $28 \mathrm{msec}$ slower for identical-noise trials than for samecategory-compatible trials with mixed presentation $[F(1,23)=12.3, p<.001]$, but not with unmixed presentation. With the latter type of presentation, there was an opposing nonsignificant 5-msec tendency toward faster responses for identical-noise trials $[F(1,23)=1.84, p>$ $.05]$. Thus, Experiment 1 replicated the previous findings that a repeated-stimulus inferiority effect is obtained when letters and digits are mixed, with a tendency toward a slight repeated-stimulus superiority effect when either letters or digits only are used.

Category relation and compatibility in mixed lists. Additional analyses were conducted on the RTs and error rates for the same- and different-category, responsecompatible and -incompatible trials in the mixed lists. These analyses showed that, as usually obtained, responses were slower and less accurate when the noise was incompatible with the target $[\mathrm{Fs}(1,23) \geq 36.5$, ps $<.001]$. Moreover, responses were slower when the category of the noise was different from that of the target $[F(1,23)=8.54, p<.008]$. Category relationship also interacted significantly with target-noise compatibility $[F(1,23)=6.15, p<.025]$, indicating that the deleterious effect of a category mismatch occurred primarily for response-compatible noise. The pattern of this deleterious effect is similar to that obtained by Flowers and Wilcox (1982) and LaBerge (1981). The effect suggests that competition between internal category responses slows responding, particularly when there is no competition between the finger responses that are signaled by the target and noise.

Summary. The results of Experiment 1 generally are consistent with the literature on comparable targetidentification tasks. Responses were slowest when the response signaled by the noise was incompatible with the response signaled by the target, indicating that response competition occurs. A small, but nonsignificant, repeatedstimulus superiority effect was obtained with unmixed presentation of letters or digits, suggesting possible competition between internal recognition responses. Similarly, with mixed presentation, responses were faster when the target and noise stimuli were from the same alphanumeric category (a category match) than when they were not (a category mismatch). The interference for category mismatches suggests that internal category responses also are generated, with competition produced when the category responses differ for target and noise. Finally, same-category-compatible trials showed a benefit over identical trials when categories were mixed, suggesting that some facilitation occurs for the former (and/or interference for the latter) in the mixed lists.

\section{EXPERIMENT 2}

In Experiment 1, the repeated-stimulus inferiority effect was obtained only for mixed lists. Experiment 2 as- sessed the possibility that the mixing of letters and digits may not be crucial for obtaining the inferiority effect. That is, in Experiment 1, as well as in all previous experiments for which more than one stimulus was assigned to each response, the unmixed lists used two stimuli per response (e.g., C. W. Eriksen \& B. A. Eriksen, 1979), whereas the mixed lists used four stimuli per response (e.g., Flowers \& Wilcox, 1982). To evaluate the relative importance of the number of stimuli versus the mixing of letters and digits for the repeated-stimulus inferiority effect, an experiment was conducted in which one group of subjects was tested with mixed presentation, in a manner similar to that used in Experiment 1, and another group was tested with unmixed presentation, but with four stimuli assigned to each response.

\section{Method}

Apparatus and Stimuli. The apparatus and stimuli were the same as in Experiment 1, with the following exceptions. The pool of letter stimuli was increased from four to eight (D, N, T, X, R, S, F, and $L)$, as was the pool of digit stimuli $(3,7,2,6,4,5,8$, and 9). For the unmixed lists, the letters were divided into two sets of four ( $D, N, T, X$, and $R, S, F, L)$ as were the digits $(3,7,2,6$, and $4,5,8,9)$. For half of the subjects who received either unmixed letters or unmixed digits, one of the sets of four was assigned to the left response and the other to the right response; for the other half, the assignments were reversed. For the mixed lists, two subsets of two letters (either $D, N$ and $R, S$ or $T, X$ and $F, L$ ) were assigned to each response, along with two subsets of two digits (either 3,7 and 4,5 or 2,6 and 8,9 ). The four combinations of letters and digits were used equally across subjects, with the assignments to the left and right responses approximately equalized.

Subjects and Procedure. Forty new subjects from the same subject pool as in Experiment 1 participated in two sessions each. Data from two additional subjects were excluded, one for having an error rate greater than $10 \%$ and the other for having RTs that exceeded 1 sec on over $10 \%$ of the trials. Each subject was tested either with mixed presentation of letters and digits or with unmixed presentation of stimuli from only one of the alphanumeric categories. The first session was regarded as practice; the second session was the test session. Except for the deviations noted, the procedure was similar to that of Experiment 1.

\section{Results and Discussion}

The mean RTs and proportions of errors are shown in Table 2. The data were analyzed in a manner similar to that used for Experiment 1.

Table 2

Mean Reaction Times (in Milliseconds) and Proportions of Errors for Experiment 2 as a Function of Presentation Method (Mixed, Unmixed) and Target-Noise Relationship

\begin{tabular}{lcccccc}
\hline & \multicolumn{6}{c}{ Target-Noise Relationship } \\
\cline { 2 - 7 } \multicolumn{1}{c}{ Measure } & I & SC & SI & N & DC & DI \\
\hline Reaction Time & 497 & 509 & 526 & 500 & & \\
Error Rate & .039 & .030 & .051 & .053 & & \\
& \multicolumn{7}{c}{ Mixed } & & & \\
Reaction Time & 545 & 531 & 546 & 523 & 536 & 552 \\
Error Rate & .022 & .028 & .063 & .047 & .024 & .040 \\
\hline
\end{tabular}

Note-I = identical $; S C=$ same-category-compatible; $S I=$ samecategory-incompatible; $N=$ neutral; $D C=$ differentcategory-compatible, and $D I=$ different-category-incompatible. 
Target-noise conditions common to mixed and unmixed lists. Two-factor analyses of the target-noise relationships common to the mixed and unmixed lists indicated that the main effects for type of list were not significant $[F s(1,38) \leq 2.62$, ps $>.05]$. Thus, when the number of alternative targets was equated for the mixed and unmixed presentations, there was no significant difference in overall difficulty between the two methods of presentation.

As in Experiment 1, both RTs and errors showed main effects for target-noise relationship $[\mathrm{Fs}(3,114) \geq 4.61$, ps $<.005]$. The slowest and least accurate responses again were on the same-category-incompatible trials (Ms $=536 \mathrm{msec}$ and .057). Unlike Experiment 1 , the neutral trials yielded the fastest responses $(\mathrm{M}=512 \mathrm{msec})$, but the error rate was high $(\mathbf{M}=.050)$. Also, the identical and same-category-compatible trials produced similar RTs (Ms $=521$ and $520 \mathrm{msec}$, respectively) and error rates $(\mathrm{Ms}=.031$ and .029$)$.

Again, an interaction of target-noise relationship with presentation type was obtained for $\operatorname{RTs}[F(3,114)=6.78$, $\mathrm{p}<.001]$. The interaction occurred because the samecategory-compatible, same-category-incompatible, and neutral conditions showed equivalent, small increases in mean RT with mixed presentation $(22,20$, and $23 \mathrm{msec}$, respectively), whereas the identical noise showed a substantial 48 -msec increase. This pattern is similar to that obtained in Experiment 1, except that the increase for the neutral condition was considerably less in the present experiment than in the previous one.

When only the identical and same-category-compatible trials were analyzed, the relationship $\times$ presentation interaction still was significant $[F(1,38)=11.5, p<.002] .^{2}$ Responses were $15 \mathrm{msec}$ slower for identical trials than for same-category-compatible trials when presentation was mixed $[F(1,19)=4,48, p<.05]$, but $12 \mathrm{msec}$ faster when presentation was unmixed, $[\mathrm{F}(1,19)=9.91, \mathrm{p}<$ $.025]$. Thus, Experiment 2 replicated the repeatedstimulus inferiority effect with mixed presentation that was obtained in Experiment 1, as well as finding a statistically reliable repeated-stimulus superiority effect with unmixed presentation. That the superiority effect obtained with unmixed presentation was statistically reliable in Experiment 2 but not in Experiment 1 may be a function of the larger target-set size used in Experiment 2.

Category relation and compatibility in mixed lists. Analyses of the same- and different-category, responsecompatible and -incompatible conditions of the mixed list again yielded compatibility main effects $[\mathrm{Fs}(1,19) \geq 10.6$, ps $<.005]$. Responses were slower and less accurate when the noise was incompatible with the response assigned to the target than when it was compatible. Unlike Experiment 1, however, there were neither main effects for category relationship (same vs. different) nor interactions of category $\times$ compatibility $[\mathrm{Fs}(1,19) \leq 3.12$, ps $>$.05]. The RT data did show a tendency for slower responses when categories differed between target and noise, but the error data showed an opposing trend. The reason why the category-relationship effect was not repli- cated in Experiment 2 is unclear. However, the effect likely is valid because it was obtained in the experiments of Flowers and Wilcox (1982) and LaBerge (1981), as well as in our Experiment 1.

Summary. The primary finding of Experiment 2 is that the repeated-stimulus inferiority effect is not due to the number of alternative targets. When the target set sizes were equated for the mixed and unmixed lists, the inferiority effect still occurred only for the mixed list. Therefore, the mixing of letters and digits seems to be necessary to obtain the repeated-stimulus inferiority effect.

\section{EXPERIMENT 3}

A statistically reliable repeated-stimulus superiority effect was obtained with unmixed presentation of letters or digits in Experiment 2, whereas only a nonsignificant trend toward such an effect was obtained in Experiment 1. One major difference between the unmixed conditions in the two experiments was that in Experiment 2 four stimuli were assigned to each response, whereas in Experiment 1 only two were. This distinction between the experiments suggests that the repeated-stimulus superiority effect is enhanced for larger target-set sizes.

Enhancement of the superiority effect with larger set sizes is predicted by the view that the effect is a function of competition between internal recognition responses (C. W. Eriksen \& Schultz, 1979). This prediction arises from the influence that increases in set size should have on the relative times for finger responses and for recognition responses. The time to identify and select the finger response signaled by the target should increase when four stimuli are assigned to each response relative to when only two are (i.e., RT generally is a positive function of targetset size; Schneider \& Shiffrin, 1977; Sternberg, 1966). However, the time to recognize the stimuli (both target and noise) should be influenced less by increases in set size, because these recognition responses have been learned and used extensively prior to the experiment. Therefore, they should be relatively automatic (Theios, $1975,1977)$. Because of the differential influence of set size on the latencies of recognition and finger responses, the internal recognition responses should precede the selection of an overt response by a longer time when the set size is large than when it is small. Thus, on samecategory-compatible trials, the opportunity for interference from the competing recognition responses should be greater with large set sizes. ${ }^{3}$

Although competition between recognition responses also should occur on same-category-incompatible trials, previous experiments have found little additional interference from competition between internal responses when the overt responses are incompatible (e.g., Flowers \& Wilcox, 1982; LaBerge, 1981; Experiment 1 of the present manuscript). This relative lack of interference from competing internal responses on incompatible trials probably is a function of the competition between overt responses overriding the lesser effects of the internal responses. Therefore, the additional interference attribut- 
able to increases in target-set size should be confined primarily to response-compatible trials.

Experiment 3 was designed to test the predicted relationship between target-set size and the magnitude of the repeated-stimulus superiority effect by varying the set size for unmixed lists. Sets of two and four targets per response were used. These set sizes correspond to those used for the unmixed conditions in Experiments 1 and 2, respectively. The finding of a larger RT advantage for identical trials would support the prediction of the competition account, and it also would serve two additional purposes. First, because the repeated-stimulus superiority effect obtained with two stimuli per response has tended to be small and not always statistically reliable (e.g., B. A. Eriksen \& C. W. Eriksen, 1974; Miller, 1982), the validity of the phenomenon should be strengthened by showing that it can be obtained reliably with a larger set size. Second, the four-target set is a more appropriate control than the two-target set for evaluating the mixing of letters and digits (because the mixed conditions of Experiments 1 and 2 used four targets per response); therefore, establishing that a substantial repeated-stimulus superiority effect is obtained reliably with the four-target set should dispell any doubts regarding the validity of the repeated-stimulus inferiority effect that is obtained with mixed presentation.

\section{Method}

Apparatus and Stimuli. The apparatus and stimuli were the same as in Experiment 2, except as noted. The four-target condition was equivalent to the unmixed condition of Experiment 2. The same subsets of either letter or digit stimuli were used, with four stimuli assigned to each of the two finger responses. For the two-target condition, only one subset of stimuli was assigned to each finger response. The subset was either $\mathrm{D}, \mathrm{N}$ and $\mathrm{R}, \mathrm{S}$, or $\mathrm{T}, \mathrm{X}$ and $\mathrm{F}, \mathrm{L}$ for letters or 3,7 and 4,5 , or 2,6 and 8,9 for digits. Within these subsets, the specific assignments of the stimulus pairs to fingers were counterbalanced across subjects.

Subjects and Procedure. Forty-eight students from the same subject pool as in Experiments 1 and 2 served as subjects. None of the subjects had participated in the previous experiments. Each subject was tested in two sessions, with the first session regarded as practice. A subject received only either the four-target condition or the two-target condition, and the same stimulus-response assignments were used for both sessions.

\section{Results and Discussion}

The mean RTs and error rates are shown in Table 3. Analyses of variance indicated significant main effects of set size (two vs. four targets assigned to each response) $[F(1,46)=7.06, p<.025]$ and target-noise relationship $[F(3,138)=22.7, p<.001]$ for RTs but not for errors (Fs $\leq 1.95$, ps $>.10]$. The main effect of set size on RT reflects slower responding when four targets were assigned to each response $(M=538 \mathrm{msec})$ than when only two were $(M=495 \mathrm{msec})$, consistent with other experiments that have varied target-set size (e.g., Schneider \& Shiffrin, 1977). The main effect for target-noise relationship is due to the usual interference for incompatible noise. The interaction of set size with target-noise relationship was not significant for either RTs or errors, although for RTs the interaction approached significance $[F(3,138)=$ $2.26, \mathrm{p}<.10]$.
Table 3

Mean Reaction Times (in Milliseconds) and Proportions of Errors for Experiment 3 as a Function of Target-Set Size and Target-Noise Relationship

\begin{tabular}{lcrrr}
\hline \multicolumn{1}{c}{ Measure } & I & SC & SI & N \\
\hline & \multicolumn{2}{c}{ Two Targets } & & \\
Reaction Time & 486 & 489 & 520 & 483 \\
Error Rate & .013 & .012 & .027 & .021 \\
& \multicolumn{2}{c}{ Four Targets } & & \\
& 521 & 541 & 555 & 535 \\
Reaction Time & 526 & .023 & .028 & .024 \\
Error Rate & .026
\end{tabular}

Note $-I=$ identical; $S C=$ same-category-compatible; $S I=$ samecategory-incompatible, and $N=$ neutral.

Despite the lack of an interaction when all target-noise conditions were considered, the RT advantage for identical trials over same-category-compatible trials was greater when four targets were assigned to each response $(20 \mathrm{msec})$ than when only two targets were $(3 \mathrm{msec})$. These differences correspond to the relative magnitudes obtained for the respective conditions in Experiments 1 and 2 , and, when only the identical and samecategory-compatible trials were analyzed, the condition $\times$ target-noise relationship interaction was significant $[F(1,46)=4.84, p<.05]$. Thus, Experiment 3 supports the implication of the previous experiments that a larger repeated-stimulus superiority effect is obtained for the identical trials when four targets are assigned to each response than when only two are.

For the reasons stated in the introduction to Experiment 3, the enhanced repeated-stimulus superiority effect obtained with four targets per response is consistent with a response-competition interpretation. According to this interpretation, RTs to same-category-compatible trials are elevated relative to those of identical-noise trials at larger set sizes because of increased competition between internal recognition responses that occurs on trials of the former type. One outcome, however, suggests some caution in accepting the competition interpretation. This outcome is that the neutral-noise trials showed a substantial increase in RT with increased set size that was comparable to that shown for the same-category-compatible trials (52 msec). If the neutral-noise condition were used as a baseline, the outcome would suggest that the samecategory-compatible trials were not showing greater interference from the competing recognition responses. However, because the neutral condition was very unstable in this series of experiments (e.g., when the unmixed two- and four-target-set conditions from Experiments 1 and 2 , respectively, are compared, the neutral condition shows the smallest increase in RT), it probably does not provide an appropriate baseline. The problem regarding the instability of the neutral-noise condition is discussed further in the General Discussion.

\section{GENERAL DISCUSSION}

The present experiments demonstrate that the repeatedstimulus superiority and inferiority effects both are 
phenomena that can be obtained reliably in the appropriate circumstances. The superiority effect occurs when the stimuli are either all letters or all digits (Experiments 1, 2 , and 3), with the small effect being enhanced by approximately $10 \mathrm{msec}$ when four stimuli, rather than two, are assigned to each response (Experiment 1 vs. 2 and Experiment 3). The repeated-stimulus inferiority effect occurs when letter and digit stimuli are mixed (Experiments 1 and 2). Thus, the present experiments substantiate the distinction implied by previous experiments (e.g., B. A. Eriksen \& C. W. Eriksen, 1974; C. W. Eriksen \& B. A. Eriksen, 1979; Flowers \& Wilcox, 1982; LaBerge, 1981) between situations that produce repeatedstimulus superiority and inferiority.

\section{Repeated Stimulus-Superiority Effect}

The repeated-stimulus superiority effect, which is obtained when all stimuli are from a single alphanumeric category, has been attributed to competition between internal recognition, or naming, responses (C. W. Eriksen \& B. A. Eriksen, 1979; C. W. Eriksen \& Schultz, 1979; Proctor \& Rao, 1983; Yeh \& C. W. Eriksen, 1984). That is, when the noise stimuli are identical to the target, the recognition responses signaled by the target and noise are in agreement, as are the overt responses. However, when the noise stimuli are different from the target but responsecompatible (i.e., assigned to the same overt response), only the overt responses are in agreement. The slower responding in the latter condition, therefore, appears to be due to the conflicting recognition responses that are indicated by the target and noise. The somewhat larger repeated-stimulus superiority effect that is obtained when four, rather than two, stimuli are assigned to each response (Experiment 3) suggests that the interference attributable to recognition-response competition is greater. As noted previously, this greater competition likely arises from the fact that the larger set size slows the selection of the overt response, but not the activation of the internal recognition responses.

Other studies have obtained several results in addition to the repeated-stimulus superiority effect that attest to the role of competition between recognition responses. When noise stimuli are ones that are not assigned to either of the possible responses, responses also are slower than when the noise is identical to the target (B. A. Eriksen \& C. W. Eriksen, 1974; Grice et al., 1984; Krueger \& Shapiro, 1980). Additionally, when pairs of letters are judged as "same" or "different," responses are slower to pairs that differ in both form and name (e.g., Bf) than to those that differ only in form (e.g., Aa). This difference is of approximately the magnitude of that obtained when one of the letters must be named (Proctor, 1981), suggesting that interference occurs from competing recognition responses when the overt response is one of sameness or difference. C. W. Eriksen \& O'Hara (1982) have obtained evidence consistent with this contention by showing that irrelevant nonsense forms interfere more with matching decisions when subjects have learned names for the forms. On the whole, the evidence for competition between recognition responses seems strong.

Experiment 1 provided evidence suggesting that similar competition also may occur between category responses when stimuli belong to distinct categories. In that experiment, responses were slower when the noise was from an alphanumeric category (letter or digit) different from that of the target, with this interference being most pronounced when the noise was response-compatible than when it was incompatible. The finding of interference from different-category noise was not replicated in Experiment 2, although a nonsignificant trend in that direction was apparent in the RT data. However, the category-interference effect is probably a valid phenomenon because it was reported by both Flowers and Wilcox (1982) and LaBerge (1981) in previous experiments that mixed letters and digits. Moreover, Shaffer and LaBerge (1979) obtained a similar category-interference effect when the stimuli were words from distinct semantic categories. Therefore, the evidence clearly suggests that category-response competition influences responding.

\section{Repeated-Stimulus Inferiority Effect}

A repeated-stimulus inferiority effect, rather than a superiority effect, occurs when letters and digits are mixed. This shift from superiority to inferiority suggests either that some interference occurs for identical noise when letters and digits are mixed that does not occur when they are unmixed or that facilitation occurs for nonidentical, response-compatible stimuli. Whether the repeatedstimulus inferiority effect should be attributed to interference or facilitation is complicated by instability for the neutral-noise condition. In Experiment 1, this control condition showed a large increase in RT from unmixed to mixed presentation that was of only slightly less magnitude than that shown for identical noise, thus supporting the facilitation interpretation. However, in Experiment 2, the neutral noise showed only a small increase, which was comparable to that shown by the samecategory-compatible noise, thus favoring the interference account. Because the neutral-noise condition was unstable across experiments, and given that other problems can arise from its use as a baseline (Jonides \& Mack, 1984; Yeh \& C. W. Eriksen, 1984), we disregarded the neutral noise when evaluating the other conditions.

Experiments 1 and 2 showed similar patterns for the effects of mixed versus unmixed presentation on the remaining three conditions. When presentation was mixed, RTs were slowed substantially more for identical-noise trials than they were for the same-category-compatible and same-category-incompatible trials (which showed comparable smaller increases). One possible interpretation of this pattern is that a benefit occurs for samecategory noise with mixed presentation, regardless of whether the response indicated by the noise is compatible or incompatible with that indicated by the target. An alternative interpretation is that mixed presentation produces interference for the identical-noise trials. This 
latter interpretation is favored by a comparison of the identical trials with the different-category-incompatible trials. Responses on the former trials were only slightly faster than those on the latter. Because, with unmixed presentation, identical trials usually show a substantial advantage over incompatible trials (the basic responsecompetition effect shown in Experiments 1-3 of the present article), the relative lack of a difference between the identical and different-category-incompatible trials with mixed presentation indicates that the identical trials are slowed relative to different-category noise, as well as relative to same-category noise. Therefore, the repeated-stimulus inferiority effect obtained with mixed presentation appears to be due to interference that occurs on the identical-noise trials.

Several possible types of explanations exist for this interference. These include explanations that attribute the interference (1) to some characteristic of the task that is correlated with the mixing of letters and digits, (2) to feature interactions, and (3) to strategies that are used when letters and digits are mixed. Several possible explanations of the first type can be generated. One correlated characteristic in previous research, as well as in our Experiment 1 , was target-set size. Although two stimuli per response were used when letters and digits were unmixed, four stimuli per response were used when letters and digits were mixed. Experiment 2 eliminated the possibility that the inferiority effect was due to the number of stimuli assigned to each response, by showing that the effect was not obtained for unmixed presentation of either letters or digits when target-set size was equivalent to that used for mixed presentation.

An additional correlated characteristic was considered in an experiment that will be described only briefly. In Experiments 1 and 2, the number (or percentage) of identical trials was half as great when presentation was mixed as when it was unmixed (this relationship also held for the same-category-compatible and samecategory-incompatible trials). The relative rarity of identical trials with mixed presentation could result in slower responses for those trials, possibly because the identity relationship would tend to attract attention. To assess this possibility, the neutral trials in the mixed lists were replaced with identical trials for some subjects, thus increasing the number of identical trials included with mixed presentation to the number included with unmixed presentation. This manipulation had no influence on the magnitude of the inferiority effect, indicating that the relative rarity of identical trials for the mixed lists is not crucial.

Another possible basis for the repeated-stimulus inferiority effect that does not rely on the category distinction is featural differences between letters and digits. For example, the category effect in visual search (faster and more accurate detection of a letter target in digits or a digit target in letters than of a letter in letters or a digit in digits) has been shown to occur primarily because there is less featural overlap between letters and digits than there is within the respective alphanumeric categories (Duncan,
1983; Krueger, 1984). However, the repeated-stimulus inferiority effect that is obtained with mixed presentation of letters and digits involves the exact same stimulus combinations within the critical target-noise conditions (i.e., the identical and same-category-compatible conditions) that are examined with unmixed presentation. Therefore, differences in featural similarity within the particular stimulus combinations for the identical and samecategory-compatible conditions cannot be the source of the effect.

Although the same-target-noise combinations are used in the mixed and unmixed lists for the crucial identical and same-category-compatible trials, the distributions of similarity values for the complete set of target-noise combinations differ. That is, because letters and digits share more features within the respective categories than between, the similarity distribution for the set of target-noise combinations is more variable when categories are mixed than when they are not. Krueger (1978) has referred to this variability as heterogeneity-of-difference. In his model of "same"-."different" judgments, heterogeneity-ofdifference acts to increase "same" RTs relative to "different' RTs (see Krueger, 1978). In the present study, the relative increase for identical trials with mixed presentation is consistent with the prediction of Krueger's model if those trials are regarded as "same" and the remaining trials as "different." Therefore, the increased heterogeneity-of-difference that results when letters and digits are mixed is a possible source of the repeatedstimulus inferiority effect. The major problem with the heterogeneity account is that there seems to be little reason why it should apply to the target-identification task in which the identity or difference of the stimuli is irrelevant to the required discrimination.

The second type of explanation for the repeated-stimulus inferiority effect involves feature interactions on identical trials. According to feature-specific inhibition models (Bjork \& Murray, 1977), responses are slower for identical-noise trials than for same-category-compatible trials because identical stimuli compete for access to the same feature analyzers. However, as noted previously, feature-specific inhibition accounts are inconsistent with the fact that the inferiority effect occurs only when letters and digits are mixed. If the interference were due to interactions that occur during feature extraction, the interference should be equally evident when letters and digits are unmixed. Therefore, if feature interactions are involved, the interactions must take place during decision processes that operate subsequent to feature extraction (e.g., Estes, 1982). The major problem with an account in terms of feature interactions is that, even if the interactions are attributed to decision-level processes, there is no obvious reason why identical features for target and noise would be more problematical when letters and digits are mixed than when they are not.

The third type of explanation attributes the repeatedstimulus inferiority effect to strategies used by subjects to perform the task. Most obviously, subjects could par- 
tition the target set according to alphanumeric category when letters and digits are mixed. Such partitioning would alter the manner in which the target set was chunked. The way in which stimuli are related to responses also might be changed. For example, one strategy that seems to be used by some subjects with unmixed presentation is to determine whether the target is assigned to one response and, if not, to make the other response. Such a strategy would appear to be less likely when letters and digits were mixed because the grouping of four digits versus four letters seems to be at least as salient as the grouping by response assignment. As with the feature-interaction account, however, the major problem with strategy accounts is the lack of a logical reason for why the strategies employed with mixed presentation, relative to those employed with unmixed presentation, would selectively interfere with identical-noise trials.

\section{Summary}

The primary contribution of the present study is to demonstrate that the repeated-stimulus superiority and inferiority effects are valid phenomena. The former is obtained when stimuli from a single alphanumeric category are used, whereas the latter is obtained when categories are mixed. Although competition between internal recognition responses is implicated as the cause of the superiority effect, the basis for the inferiority effect is unclear. The most likely possible explanations involve the similarity distributions for the target-noise stimulus combinations, decision-level feature interactions, or the strategies used to relate the stimuli to responses. However, at present, none of these explanations provides an adequate account of the effect. The repeated-stimulus inferiority effect is an intriguing, potentially insightful phenomenon whose nature, we hope, will be clarified in future research.

\section{REFERENCES}

Bjork, E. L., \& Murray, J. T. (1977). On the nature of input channels in visual processing. Psychological Review, 84, 472-484.

Crosland, H. R. (1931). Letter-position effects in the range of attention experiment, as affected by the number of letters in each exposure. Journal of Experimental Psychology, 14, 477-507.

Duncan, J. (1983). Category effects in visual search: A failure to replicate the "oh-zero" phenomenon. Perception \& Psychophysics, 34, 221-232.

ERIKSEN, B. A., \& ERIKSEN, C. W. (1974). Effects of noise letters upon the identification of a target letter in a nonsearch task. Perception \& Psychophysics, 16, 143-149.

ERIKSEN, C. W. (1966). Independence of successive inputs and uncorrelated error in visual form perception. Journal of Experimental Psychology, 72, 26-35.

ERIKSEN, C. W., \& ERIKSEN, B. A. (1979). Target redundancy in visual search: Do repetitions of the target within the display impair processing? Perception \& Psychophysics, 26, 195-205.

Eriksen, C. W., Morris, N., Yeh, Y.-Y., O'Hara, W., \& Durst, R. T. (1981). Is recognition accuracy really impaired when the target is repeated in the display? Perception \& Psychophysics, 30, 375-385.

ERIKSEN, C. W. \& O'HARA, W. P. (1982). Are nominal same-different matches slower due to differences in level of processing or to response competition? Perception \& Psychophysics, 32, 335-344.

ERIKSEN, C. W., \& Schultz, D. W. (1979). Information processing in visual search: A continuous flow conception and experimental results. Perception \& Psychophysics, 25, 249-263.

EsTEs, W. K. (1982). Similarity-related channel interactions in visual processing. Joumal of Experimental Psychology: Human Perception and Performance, 8, 353-382.

Flowers, J. H. (1985). Priming effects in perceptual classification. Unpublished manuscript.

Flowers, J. H., \& Wilcox, N. (1982). The effect of flanking context on visual classification: The joint contribution of interactions at different processing levels. Perception \& Psychophysics, 32, 581-591.

Grice, G. R., Boroughs, J. M., \& Canham, L. (1984). Temporal dynamics of associative interference and facilitation produced by visual context. Perception \& Psychophysics, 36, 499-507.

Grice, G. R., Canham, L., \& Schafer, C. (1982). Development of associative and perceptual interference. Perception \& Psychophysics, 32, 375-387.

GricE, G. R., GWYNNE, J. W. (1985). Temporal characteristics of noise conditions producing facilitation and interference. Perception \& Psychophysics, 37, 495-501.

JoNides, J., \& MACK, R. (1984). On the cost and benefit of cost and benefit. Psychological Bulletin, 96, 29-44.

Krueger, L. E. (1978). A theory of perceptual matching. Psychological Review, 85, 278-304.

KRUEGER, L. E. (1984). The category effect in visual search depends on physical rather than conceptual differences. Perception \& Psychophysics, 35, 558-564.

Krueger, L. E., \& Shapiro, R. G. (1980). Repeating the target neither speeds nor slows its detection: Evidence for independent channels in letter processing. Perception \& Psychophysics, 28, 68-76.

LABERGE, D. (1981). Unitization and automaticity in perception. In J. H. Flowers (Ed.), 1980 Nebraska Symposium on Motivation: Cognitive Processes (Vol. 28, pp. 53-71). Lincoln: University of Nebraska Press.

La Heis, W., \& van der Heidden, A. H. C. (1983). Feature-specific interference in letter identification. Acta Psychologica, 53, 37-60.

MilleR, J. (1982). Effects of noise letters on decisions: Discrete or continuous flow of information? Perception \& Psychophysics, 31, 227-236.

Proctor, R. W. (1981). A unified theory for matching-task phenomena. Psychological Review, 88, 291-326.

Proctor, R. W., RAO, K. V. (1983). Reinstating the original principles of Proctor's unified theory for matching-task phenomena: An evaluation of Krueger and Shapiro's reformulation. Psychological Review, 90, 21-37.

SANTEE, J. L., \& EGTH, H. E. (1980). Interference in letter identification: A test of feature-specific inhibition. Perception \& Psychophysics, 27, 321-330

SANTEE, J. L., \& EGETH, H. E. (1982). Do reaction time and accuracy measure the same aspects of letter recognition? Joumal of Experimental Psychology: Human Perception and Performance, 8, 489-501.

SChNEIDER, W., \& SHIFfrin, R. M. (1977). Controlled and automatic human information processing: I. Detection, search, and attention. Psychological Review, 84, 1-66.

ShafFer, W. O., \& LABERGe, D. (1979). Automatic semantic processing of unattended words. Joumal of Verbal Learning and Verbal Behavior, $18,413-426$.

STERNBERG, S. (1966). High-speed scanning in human memory. Science, $153,652-654$.

TAYLOR, D. A. (1977). Time course of context effects. Journal of Experimental Psychology: General, 106, 404-426.

THEIOS, J. (1975). The components of response latency in simple human information processing tasks. In P. M. A. Rabbitt \& S. Dornic (Eds.), Attention and Performance, $V$ (pp. 418-440). New York: Academic Press.

Theios, J. (1977). Commentary on "Reaction time measurements in the study of memory processes: Theory and data." In G. H. Bower 
(Ed.), Human memory: Basic processes (pp. 253-257). New York: Academic Press.

YEH, Y.-Y., \& ERIKSEN, C. W. (1984). Name codes and features in the discrimination of letter forms. Perception \& Psychophysics, 36, 225-233

\section{NOTES}

1. Flowers (1985) recently replicated the repeated-stimulus inferiority effect with a different set of letters and digits. Both his study and Flowers and Wilcox's (1982) indicate that the inferiority for identical-noise trials occurs primarily when the noise and target are presented simultaneously. With successive presentation, the inferiority effect is obtained only if the interval between the noise and target onsets is less than $200 \mathrm{msec}$.
2. The error data showed an opposing, though nonsignificant, pattern to the RT data for the identical and same-category-incompatible conditions. This relationship was not obtained in our other experiments, so the RT data likely do not reflect a speed-accuracy tradeoff.

3. Although Experiment 3 used only unmixed lists in which differentcategory noise does not occur, the labels "same-category-compatible" and "same-category-incompatible" have been retained to be consistent with the terminology used for Experiments 1 and 2.

(Manuscript received June 3, 1985; revision accepted for publication August 5, 1985.) 